\title{
Competitive Two-Agent Scheduling with Learning Effect and Release Times on a Single Machine
}

\author{
Der-Chiang $\mathrm{Li}^{1}$ and Peng-Hsiang Hsu ${ }^{1,2}$ \\ ${ }^{1}$ Department of Industrial and Information Management, National Cheng Kung University, No. 1, \\ University Road, Tainan 70101, Taiwan \\ ${ }^{2}$ Department of Business Administration, Kang-Ning Junior College, Taipei, Taiwan
}

Correspondence should be addressed to Peng-Hsiang Hsu; r38991036@mail.ncku.edu.tw

Received 16 May 2013; Accepted 2 July 2013

Academic Editor: Yunqiang Yin

Copyright (c) 2013 D.-C. Li and P.-H. Hsu. This is an open access article distributed under the Creative Commons Attribution License, which permits unrestricted use, distribution, and reproduction in any medium, provided the original work is properly cited.

\begin{abstract}
The learning effect has gained much attention in the scheduling research recently, where many researchers have focused their problems on only one optimization. This study further addresses the scheduling problem in which two agents compete to perform their own jobs with release times on a common single machine with learning effect. The aim is to minimize the total weighted completion time of the first agent, subject to an upper bound on the maximum lateness of the second agent. We propose a branchand-bound approach with several useful dominance properties and an effective lower bound for searching the optimal solution and three simulated-annealing algorithms for the near-optimal solutions. The computational results show that the proposed algorithms perform effectively and efficiently.
\end{abstract}

\section{Introduction}

In traditional scheduling problems, most studies assumed that each of the operations of all job processing times is known and fixed. But in some circumstances, the jobs processing times are affected by learning effect. The "learning effect" is the phenomenon that unit costs reduce as firms produce more of a product and gain knowledge or experience. Biskup [1] and Cheng and Wang [2] first brought the phenomenon of learning effect into scheduling field. Afterwards the learning effect is getting much to pay attention in the scheduling research in the last decade such as Mosheiov [3], Mosheiov and Sidney [4], Bachman and Janiak [5], Lee and Wu [6], Kuo and Yang [7], and Koulamas and Kyparisis [8]. Biskup [9] provided the most recent learning effects survey paper in scheduling research. More recent studies involving learning effects were by Wang et al. [10], Janiak and Rudek [11], Yin et al. [12], Toksari and Güner [13], Wang et al. [14], Lee et al. [15], J.-B. Wang and C. Wang [16], Wu et al. [17], Zhang et al. [18], Yin et al. [19], and Yang et al. [20], Yang et al. [21], Wang et al. [22], J.-B. Wang and J.-J. Wang [23], Cheng et al. [24], and so forth.
In addition, most research assumed that all jobs met a single criterion. But jobs might come from different agents. There might be multiple agents who compete on the same resources, and each agent has its own objective. This concept was first initiated and considered into scheduling field by Baker and Smith [25] and Agnetis et al. [26]. After that time, many researchers focused on multiagent in scheduling field. However, little research has been done on scheduling problem with learning effect and multiagent. Liu et al. [27] studied the optimal polynomial time algorithms to solve a singlemachine scheduling problem with two-agent and positiondependent processing time aging and learning effect. The objective is to find a schedule that minimizes the total completion time of the first agent with a maximum cost limit of the second agent. Cheng et al. [28] investigated a twoagent single-machine scheduling problem with a truncated sum-of-processing times-based learning effect and developed algorithms to minimize the total weighted completion time of the jobs of the first agent, subject to the restriction that no tardy job is allowed for the second agent. Li and Hsu [29] investigated the job scheduling problem of two agents competing for the usage of a common single machine with 
learning effect. The objective is to minimize the total weighted completion time of both agents with the restriction that the makespan of either agent cannot exceed an upper bound. Wu et al. [30] address a two-agent single-machine scheduling problem with the co-existing sum-of-processing times-based deteriorating and learning effects. The goal is to minimize the total weighted completion time of the jobs of the first agent given that no tardy job is allowed for the second agent.

The previous both scheduling issues were yet relatively unexplored. In this paper, we therefore study a two-agent scheduling problem with position-based learning competing on a common single machine and further consider each job a different release time. The objective is to minimize the total weighted completion time of first agents, subject to the constraint that the maximum lateness of second agent the jobs cannot exceed an upper bound. In the classical scheduling notation, the problem can be notated by a triplet as $1\left|r_{j}^{X}, p_{j k}^{X}=p_{j}^{X} k^{a}\right| \sum w_{j}^{A} C_{j}^{A}: L_{\max }^{B} \leq Q$. As shown by Agnetis et al. [26], it is a Binary NP-hard problem, even without release time and learning effect $\left(1 \| \sum w_{j}^{A} C_{j}^{A}: f_{\max }^{B}\right)$.

The rest of this paper is organized as follows: the problem formulation is introduced in the next section. The branch-and-bound and simulated-annealing algorithms are employed to find the optimal solution and the near-optimal solutions, respectively. Dominance properties and a lower bound are developed to be used in the branch-and-bound algorithm in Section 3. The details of simulated-annealing algorithm are described in Section 4, and the computational experiment results are given in Section 5. In the last section, some conclusions and extensions are presented.

\section{Problem Formulation}

In this section, we describe a formal definition of the model as there are $n$ jobs from two competing agents ( $X=$ agent $A$ or agent $B$ ) to be scheduled. The number of jobs in the two sets is recorded as $n^{A}$ and $n^{B}$, such that $n=n^{A}+n^{B}$. The processing time and weight of job $j$ are known and denoted as $p_{j}^{X}$ and $w_{j}^{X}$, respectively. Based on the learning effect, the actual processing time $p_{j k}^{X}$ of job $j$ changes with position $k$ and learning ratio $a$, that is $p_{j k}^{X}=p_{j}^{X} k^{a}$, where $a<0$ and $k=1,2, \ldots, n$.

Furthermore, we consider the problem of scheduling a set $S$ of $n$ independent jobs with integer release times $\left(r_{j}^{X}\right)$ on single machine. The goal is to minimize the weighted sum of completion times of the jobs from agent $A$, subject to the constraint that the maximum lateness of the jobs from agent $B$ is not more than a given upper bound $Q$.

\section{Branch-and-Bound Algorithm}

The computational complexity of this problem does not consider ready time and learning effect $\left(1 \| \sum w_{j} C_{j}^{A}: f_{\max }^{B}\right)$, which is showed a Binary NP-hard problem by Agnetis et al. [26]. Therefore, the addressed problem here $\left(1 \mid r_{j}^{X}, p_{j k}^{X}=\right.$ $\left.p_{j}^{X} k^{a} \mid \sum w_{j} C_{j}^{A}: L_{\max }^{B}\right)$ is more complex than the problem
(1 $\| \sum w_{j} C_{j}^{A}: f_{\max }^{B}$ ). We basically try to employ the branch-and-bound algorithm to gain the optimal solution, and for speeding up the searching process, several dominance properties and a lower bound were presented in the following.

3.1. Dominance Property. Suppose that there are two contiguous jobs $\left(J_{i}^{X}\right.$ and $\left.J_{j}^{X}\right)$ in sequence $S_{1}=\left(\pi, J_{i}^{X}, J_{j}^{X}, \pi^{c}\right)$, where $\pi$ and $\pi^{c}$ denote the scheduled and unscheduled partial sequence, respectively. One can perform the contiguous jobs $J_{i}^{X}$ and $J_{j}^{X}$ interchanges to obtain another sequence $S_{2}=\left(\pi, J_{j}^{X}, J_{i}^{X}, \pi^{c}\right)$. To show that $S_{1}$ dominates $S_{2}$, it is sufficient to ensure $C_{j}^{X}\left(S_{1}\right) \leq C_{i}^{X}\left(S_{2}\right)$ or $\left[w_{i}^{X} C_{i}^{X}\left(S_{1}\right)+\right.$ $\left.w_{j}^{X} C_{j}^{X}\left(S_{1}\right)\right] \leq\left[w_{i}^{X} C_{i}^{X}\left(S_{2}\right)+w_{j}^{X} C_{j}^{X}\left(S_{2}\right)\right]$. Before proving the proposed properties, we let $t$ denote the completion time of the last job in the scheduled partial sequence $\pi$ to determine the following properties.

Property 1. If $J_{i}^{X}, J_{j}^{X} \in J^{A}, \max \left\{r_{j}^{A}, t\right\} \geq \max \left\{r_{i}^{A}, t\right\}, p_{i}^{A} \leq p_{j}^{A}$, and $w_{i}^{A}>w_{j}^{A}$, then $S_{1}$ dominates $S_{2}$.

Proof. By the definition, the completion times of jobs $J_{i}^{A}$ and $J_{j}^{A}$ in $S_{1}$ and $S_{2}$ are, respectively,

$$
\begin{aligned}
C_{i}^{A}\left(S_{1}\right) & =\max \left\{r_{i}^{A}, t\right\}+p_{i}^{A} k^{a}, \\
C_{j}^{A}\left(S_{1}\right) & =\max \left\{\max \left\{r_{i}^{A}, t\right\}+p_{i}^{A} k^{a}, r_{j}^{A}\right\}+p_{j}^{A}(k+1)^{a}, \\
C_{j}^{A}\left(S_{2}\right) & =\max \left\{r_{j}^{A}, t\right\}+p_{j}^{A} k^{a}, \\
C_{i}^{A}\left(S_{2}\right) & =\max \left\{\max \left\{r_{j}^{A}, t\right\}+p_{j}^{A} k^{a}, r_{i}^{A}\right\}+p_{i}^{A}(k+1)^{a} \\
& =\max \left\{r_{j}^{A}, t\right\}+p_{j}^{A} k^{a}+p_{i}^{A}(k+1)^{a} .
\end{aligned}
$$

Since $\max \left\{r_{j}^{A}, t\right\} \geq \max \left\{r_{i}^{A}, t\right\}$ and $p_{i}^{A} \leq p_{j}^{A}, C_{j}^{A}\left(S_{1}\right) \leq$ $C_{i}^{A}\left(S_{2}\right)$. Moreover,

$$
\begin{aligned}
& w_{j}^{A} C_{j}^{A}\left(S_{2}\right)+w_{i}^{A} C_{i}^{A}\left(S_{2}\right)-\left(w_{i}^{A} C_{i}^{A}\left(S_{1}\right)+w_{j}^{A} C_{j}^{A}\left(S_{1}\right)\right) \\
&=w_{j}^{A}\left(\max \left\{r_{j}^{A}, t\right\}+p_{j}^{A} k^{a}\right) \\
&+w_{i}^{A}\left(\max \left\{r_{j}^{A}, t\right\}+p_{j}^{A} k^{a}+p_{i}^{A}(k+1)^{a}\right) \\
&-w_{i}^{A}\left(\max \left\{r_{i}^{A}, t\right\}+p_{i}^{A} k^{a}\right) \\
&-w_{j}^{A}\left(\max \left\{\max \left\{r_{i}^{A}, t\right\}+p_{i}^{A} k^{a}, r_{j}^{A}\right\}+p_{j}^{A}(k+1)^{a}\right) \\
& \geq w_{j}^{A}\left(\max \left\{r_{j}^{A}, t\right\}+p_{j}^{A} k^{a}\right) \\
&+w_{i}^{A}\left(\max \left\{r_{j}^{A}, t\right\}+p_{j}^{A} k^{a}+p_{i}^{A}(k+1)^{a}\right) \\
&-w_{i}^{A}\left(\max \left\{r_{i}^{A}, t\right\}+p_{i}^{A} k^{a}\right) \\
&-w_{j}^{A}\left(\max \left\{r_{i}^{A}, t\right\}+p_{i}^{A} k^{a}+p_{j}^{A}(k+1)^{a}\right) \\
&=\left(w_{i}^{A}+w_{j}^{A}\right)\left(\max \left\{r_{j}^{A}, t\right\}-\max \left\{r_{i}^{A}, t\right\}\right)
\end{aligned}
$$




$$
\begin{aligned}
& +w_{j}^{A} p_{j}^{A} k^{a}+w_{i}^{A}\left(p_{j}^{A} k^{a}+p_{i}^{A}(k+1)^{a}\right) \\
& -w_{i}^{A} p_{i}^{A} k^{a}-w_{j}^{A}\left(p_{i}^{A} k^{a}+p_{j}^{A}(k+1)^{a}\right) .
\end{aligned}
$$

By $p_{i}^{A} \leq p_{j}^{A}$ and $w_{i}^{A}>w_{j}^{A}$, we have

$$
\begin{aligned}
& w_{j}^{A} p_{j}^{A} k^{a}+w_{i}^{A}\left(p_{j}^{A} k^{a}+p_{i}^{A}(k+1)^{a}\right) \\
& -w_{i}^{A} p_{i}^{A} k^{a}-w_{j}^{A}\left(p_{i}^{A} k^{a}+p_{j}^{A}(k+1)^{a}\right)>0,
\end{aligned}
$$

hence $\left[w_{i}^{A} C_{i}^{A}\left(S_{1}\right)+w_{j}^{A} C_{j}^{A}\left(S_{1}\right)\right] \leq\left[w_{i}^{A} C_{i}^{A}\left(S_{2}\right)+w_{j}^{A} C_{j}^{A}\left(S_{2}\right)\right]$, as required.

The proofs of Properties 2-4 are omitted since they are similar to that of Property 1.

Property 2. If $J_{i}^{X}, J_{j}^{X} \in J^{A}, r_{j}^{A} \geq \max \left\{r_{i}^{A}, t\right\}+p_{i}^{A} k^{a}$, and $p_{i}^{A} \leq$ $p_{j}^{A}$, then $S_{1}$ dominates $S_{2}$.

Property 3. If $J_{i}^{X}, J_{j}^{X} \in J^{B}, \max \left\{\max \left\{r_{i}^{B}, t\right\}+p_{i}^{B} k^{a}, r_{j}^{B}\right\}+$ $p_{j}^{B}(k+1)^{a}-d_{j}^{B} \leq 0$, and $\max \left\{r_{i}^{B}, t\right\}+p_{i}^{B} k^{a}-d_{i}^{B} \leq 0<$ $\max \left\{\max \left\{r_{j}^{B}, t\right\}+p_{j}^{B} k^{a}, r_{i}^{B}\right\}+p_{i}^{B}(k+1)^{a}-d_{i}^{B}$, then $S_{1}$ dominates $S_{2}$.

Property 4. If $J_{i}^{X} \in J^{A}, J_{j}^{X} \in J^{B}, \max \left\{r_{j}^{B}, t\right\} \geq \max \left\{r_{i}^{A}, t\right\}$, $p_{i}^{A} \leq p_{j}^{B}$, and $\max \left\{\max \left\{r_{i}^{A}, t\right\}+p_{i}^{A} k^{a}, r_{j}^{B}\right\}+p_{j}^{B}(k+1)^{a}-d_{j}^{B} \leq Q$, then $S_{1}$ dominates $S_{2}$.

In addition, let $\left(\pi, \pi^{c}\right)$ be a sequence of the jobs where $\pi$ is the scheduled part with $k$ jobs, and $\pi^{c}$ is the unscheduled part with $(n-k)$ jobs. The following property is found for determining the sequence feasibility by the unscheduled $J_{j}^{B}$. Moreover, $C_{[k]}$ is the completion time of the last job in $\pi$.

Property 5. If there is a $J_{j}^{B}$ in $\pi^{c}$ such that $\max \left\{C_{[k]}, r_{j}^{B}\right\}+$ $p_{j}^{B} n^{a}-d_{j}^{B}>Q$, then sequence $\left(\pi, \pi^{c}\right)$ is not a feasible solution.

Proof. Since $\max \left\{C_{[k]}, r_{j}^{B}\right\}+p_{j}^{B} n^{a}-d_{j}^{B}>Q$, lateness of the unscheduled jobs $J_{j}^{B}$ must exceed the given bound $Q$. So $\left(\pi, \pi^{c}\right)$ is not a feasible sequence.

The next property is for assigning the unscheduled $J_{j}^{A}$ in $(k+1)$ th position.

Property 6. If all the unscheduled jobs belong to $J^{A}$ and there exists an $J_{j}^{A}$ such that $\max \left\{C_{[k]}, r_{j}^{A}\right\}+p_{j}^{A}(k+1)^{a} \leq r^{*}$, where $r^{*}=\min \left\{r_{i}^{A}\right\}$ for all jobs $J_{i}^{A} \in \pi^{c}$ and $J_{i}^{A} \neq J_{j}^{A}$, then job $J_{j}^{A}$ may be assigned to the $(k+1)$ th position.

3.2. Lower Bound. In addition to the previous properties, we hatch up a lower bound to speed up the building of searching trees in the branch-and-bound algorithm. Assume that PS and US are the two partial scheduling sequences. PS is the scheduled $k$ jobs, and US is the remaining $(n-k)$ unscheduled jobs in which $n_{1}$ is agent $A$ jobs and $n_{2}$ is agent $B$ jobs, where $n_{1}+n_{2}=n-k$. The lower bound is obtained by scheduling agent $A$ jobs first and then scheduling agent $B$ jobs in any order. To elaborate this, let $C_{[k]}^{X}$ be the completion time of the last job in PS; then the completion time for the $(k+1)$ th job is

$$
\begin{aligned}
C_{[k+1]}^{A}(S) & =\max \left\{C_{[k]}^{X}(S), r_{[k+1]}^{A}\right\}+p_{[k+1]}^{A}(k+1)^{a} \\
& \geq r_{[k+1]}^{A}+p_{[k+1]}^{A}(k+1)^{a} \\
& \geq r_{[k+1]}^{A}+p_{[k+1]}^{A} n^{a} .
\end{aligned}
$$

Similarly, the completion time for the $(k+l)$ job is

$$
\begin{aligned}
C_{[k+l]}^{A}(S) & =\max \left\{C_{[k+l-1]}^{A}(S), r_{[k+l]}^{A}\right\}+p_{[k+l]}^{A}(k+l)^{a} \\
& \geq r_{[k+l]}^{A}+p_{[k+l]}^{A} n^{a}, \quad 2 \leq l \leq n_{1} .
\end{aligned}
$$

Hence the lower bound of the partial sequence PS can thus be found as follows:

$$
\mathrm{LB}=\sum_{J_{j}^{A} \in \mathrm{AS}} w_{j}^{A} C_{j}^{A}+n^{a} \sum_{J_{j}^{A} \in \mathrm{US}} w_{j}^{A}\left(r_{j}^{A}+p_{j}^{A}\right) .
$$

\section{Simulated-Annealing Algorithm}

The simulated-annealing algorithm is one of the metaheuristic methods to solve large-scaled combinatorial minimization problems $[28,31-34]$. It was first described by Kirkpatrick et al. [35] based upon the research of Metropolis et al. [36]. The major advantage of this approach is that it avoids getting trapped in local minima for global optimization by controlling the parameter which influences the probability of accepting a worse solution in the iterative process. Here, we employ SA algorithm to obtain near-optimal solutions as described in the following.

Step 1 (initial feasible solution). An initial feasible sequence was generated by putting agent $B$ in front of agent $A$ for considering the conditionality objective. Thus, we employ the earliest due date (EDD) rule for agent $B$ first, and for agent $A$, four different rules are employed as EDD rule, shortest processing time (SPT) rule, shortest release time (SRT) rule, and weighted shortest processing time (WSPT) rule; they were denoted by $\mathrm{SA}_{1}, \mathrm{SA}_{2}, \mathrm{SA}_{3}$, and $\mathrm{SA}_{4}$, respectively.

Step 2 (adjusting the solution). To improve on the initial schedule, we shift the neighborhood job schedules. The exchange sequence strategy procedures were to choose two different locations randomly and irregularly select one of the three resources (pairwise interchanges, extraction, and forward/backward shifted reinsertions) to ameliorate the quality of the SA.

Step 3 (acceptance probability). If there exists a new schedule that improves the value of the objective, it replaces the previous schedule. Besides, the SA algorithms prevent it to get stuck to local minima with an acceptance probability. The acceptance probability, given in the next equation, is based on the exponential distribution:

$$
P(\text { accept })=\exp (-\lambda \times \Delta \mathrm{WC}),
$$


where $\lambda$ is the control parameter and $\Delta \mathrm{WC}$ is the variation of the objective value. If $P($ accept $)>\operatorname{rand}(0,1)$, the new sequence is accepted, otherwise new sequence will be rejected. Ben-Arieh and Maimon [37] suggested that $\lambda$ in the $k$ th iteration is

$$
\lambda=\frac{k}{\beta}
$$

where $\beta$ is an experimental constant. After preliminary trials, $\beta=2$ was used in our experiment.

4.1. Stopping Condition Iterations. The SA algorithms were terminated after $300 n$ iterations in our preliminary experiments, where $n$ is the number of jobs.

\section{Computational Experiments}

A computational experiment was conducted to assess the performance of the proposed branch-and-bound algorithm and the accuracy of the SA algorithms. All the algorithms were coded in Compaq Visual Fortran version 6.6 and run on an Intel(R) Core(TM) i7-2600 CPU @ 3.40 GHz with 4 GB RAM operating system under Windows 7 environment. The experimental design followed Chu's [38] and Fisher [39] framework, where the normal job processing times were generated randomly from a uniform distribution over the integers between 1 and 100; the release times were from a uniform distribution over the integers $(0,50.5 n \lambda)$, where $n$ was the job size and $\lambda$ was a control variable; the due dates were from a uniform distribution over the range of integers $T(1-\tau-\gamma / 2)$ to $T(1-\tau+\gamma / 2)$, where $T$, $\tau$, and $\gamma$ are the sum of the processing times of all the jobs $T=\sum_{i=1}^{n} p_{i}$, the tardiness factor, and the due date range, respectively. Furthermore, the bound $Q$ was fixed at 0 and each agent has half of the total number of jobs.

5.1. The Accuracy of SAs for Small Job Size. First, to assess the accuracy of SA algorithms, the error percentage was calculated as

$$
\frac{\left(\mathrm{SA}_{i}-\mathrm{OP}\right)}{\mathrm{OP}} \times 100 \%,
$$

where $\mathrm{SA}_{i}(i=1,2,3,4,5)$ was the solution obtained from the SA algorithm and OP was the optimal solution of the objective function obtained from the branch-and-bound algorithm. In the first simulation experiment, the job size $(n)$, tardiness factor $(\tau)$, the due date range $(\gamma)$, and learning effect (le) were fixed at $(n, \tau, \gamma$, le $)=(12,0.25,0.25,80)$. The values of $\lambda$ were taken as $0.2,0.4,0.6,0.8,1.0,1.25,1.5,1.75,2.0$, and 3.0. For each situation, 100 replications were randomly generated. The mean and maximum of the error percentages were recorded in Table 1 . The results showed that $\mathrm{SA}_{1}, \mathrm{SA}_{2}$, $\mathrm{SA}_{3}$, and $\mathrm{SA}_{4}$ are not affected by the variation of $\lambda$. It was observed that the mean error percentage of the SA algorithm was less than $0.1352 \%$. In order to diminish some peculiar worst case of SAs, we combined the four SAs to obtain $\mathrm{SA}_{5}$ which was the smallest value of the objective function

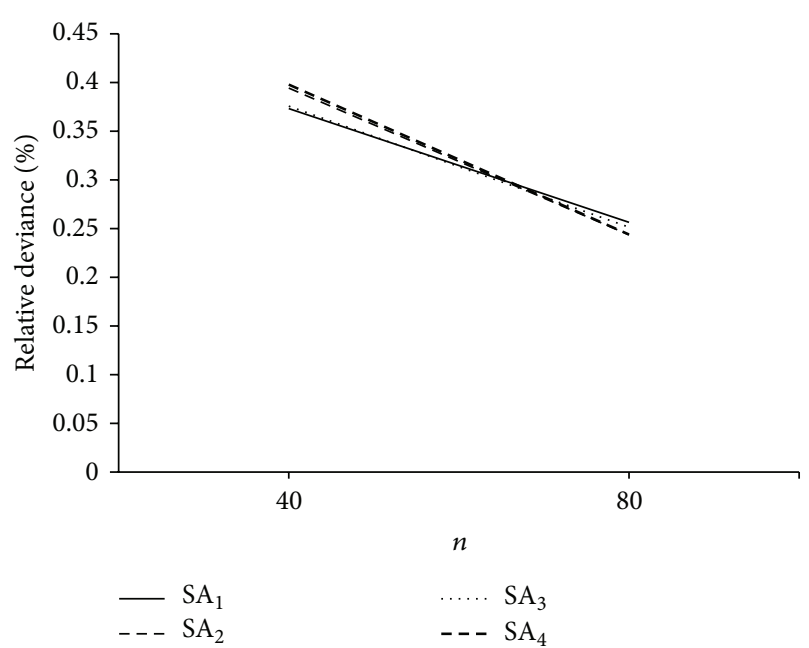

FIGURE 1: The performance of SA algorithm with respect to $n$.

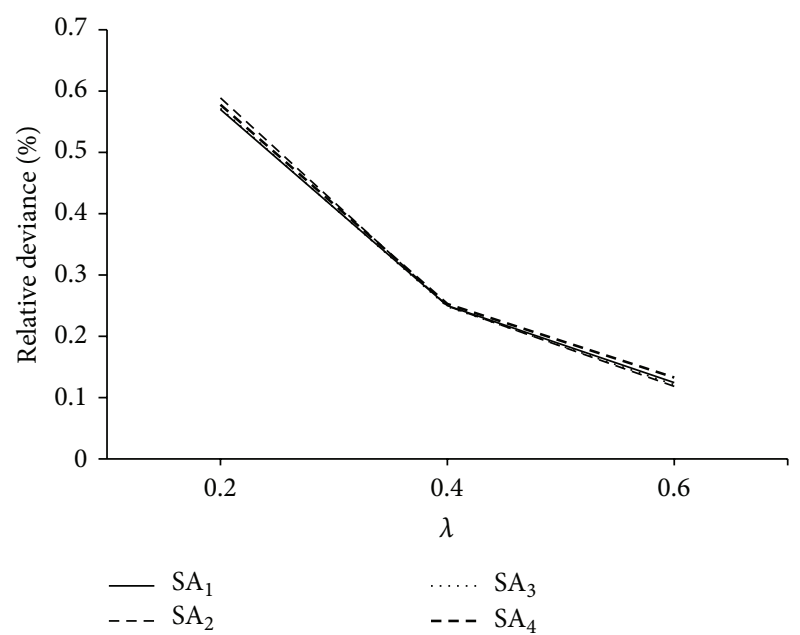

Figure 2: The performance of SA algorithm with respect to $\lambda$.

obtained from the SAs. The mean error percentage of the $\mathrm{SA}_{5}$ was less than $0.0429 \%$.

In another simulation experiment, variables were fixed at $(n, \lambda, \mathrm{le})=(12,0.4,80)$. The values of $(\tau, \gamma)$ were taken as $(0.25,0.25),(0.25,0.5),(0.25,0.75),(0.5,0.25),(0.5,0.5)$, and $(0.5,0.75)$. For each situation, 100 replications were randomly generated. The mean and maximum of the percentage errors were also recorded in Table 2 . The results were similar to the former experiment. The mean error percentage of the $\mathrm{SA}_{5}$ was less than $0.0151 \%$. By the two experiment results, it was recommended to combine $\mathrm{SAs}$ into $\mathrm{SA}_{5}$.

5.2. Performance of the Branch-and-Bound Algorithm for Small Job Size. To evaluate the performance of the branchand-bound algorithm, the mean and maximum numbers of nodes were recorded as well as the computation times (in seconds) to calculate the mean and maximum computation times. In this simulation experiment, the parameters were set as the job size ( $n=12$ and 14$)$, release times control value 
TABLE 1: The performance of the simulated-annealing algorithms at $(n, \tau, \gamma, \mathrm{le})=(12,0.25,0.25,80)$.

\begin{tabular}{lccccccccccc}
\hline$\lambda$ & \multicolumn{2}{c}{$\mathrm{SA}_{1}$} & \multicolumn{2}{c}{$\mathrm{SA}_{2}$} & \multicolumn{2}{c}{$\mathrm{SA}_{3}$} & \multicolumn{2}{c}{$\mathrm{SA}_{4}$} & \multicolumn{2}{c}{$\mathrm{SA}_{5}$} \\
& Mean & Max & Mean & Max & Mean & Max & Mean & Max & Mean & Max \\
\hline 0.20 & 0.0252 & 0.6711 & 0.0326 & 1.2731 & 0.0294 & 1.2731 & 0.0239 & 1.2731 & 0.0020 & 0.2014 \\
0.40 & 0.0539 & 1.9013 & 0.0724 & 1.9013 & 0.0911 & 1.9013 & 0.1352 & 5.8067 & 0.0151 & 1.4364 \\
0.60 & 0.0433 & 0.9016 & 0.0954 & 5.5725 & 0.0803 & 4.4405 & 0.0919 & 4.6113 & 0.0103 & 0.3001 \\
0.80 & 0.0595 & 1.8690 & 0.0412 & 0.4131 & 0.0465 & 1.6664 & 0.0543 & 1.7206 & 0.0062 & 0.1241 \\
1.00 & 0.0710 & 3.7936 & 0.1013 & 3.7936 & 0.0994 & 3.7936 & 0.0907 & 3.7936 & 0.0429 & 3.7936 \\
1.25 & 0.0423 & 0.2853 & 0.1068 & 5.7572 & 0.0433 & 0.4495 & 0.0371 & 0.6049 & 0.0045 & 0.0665 \\
1.50 & 0.0359 & 0.4688 & 0.0414 & 0.2745 & 0.0331 & 0.3646 & 0.0331 & 0.2364 & 0.0048 & 0.0651 \\
1.75 & 0.0671 & 3.3128 & 0.0414 & 0.3602 & 0.0671 & 3.3128 & 0.0413 & 0.3486 & 0.0057 & 0.1112 \\
2.00 & 0.0358 & 0.3843 & 0.0329 & 0.3610 & 0.0504 & 0.3250 & 0.0314 & 0.3368 & 0.0091 & 0.2887 \\
3.00 & 0.0355 & 0.2558 & 0.0420 & 0.2762 & 0.0321 & 0.2738 & 0.0316 & 0.3117 & 0.0066 & 0.1416 \\
\hline
\end{tabular}

TABLE 2: The performance of the simulated-annealing algorithms at $(n, \lambda, l e)=(12,0.4,80)$.

\begin{tabular}{lcccccccccccc}
\hline$\tau$ & \multicolumn{2}{c}{$\mathrm{SA}_{1}$} & \multicolumn{2}{c}{$\mathrm{SA}_{2}$} & \multicolumn{2}{c}{$\mathrm{SA}_{3}$} & \multicolumn{2}{c}{$\mathrm{SA}_{4}$} & \multicolumn{2}{c}{$\mathrm{SA}_{5}$} \\
& $\gamma$ & Mean & Max & Mean & Max & Mean & Max & Mean & Max & Mean & Max \\
\hline \multirow{2}{*}{0.25} & 0.25 & 0.0252 & 0.6711 & 0.0326 & 1.2731 & 0.0294 & 1.2731 & 0.0239 & 1.2731 & 0.0020 & 0.2014 \\
& 0.50 & 0.0539 & 1.9013 & 0.0724 & 1.9013 & 0.0911 & 1.9013 & 0.1352 & 5.8067 & 0.0151 & 1.4364 \\
& 0.75 & 0.0433 & 0.9016 & 0.0954 & 5.5725 & 0.0803 & 4.4405 & 0.0919 & 4.6113 & 0.0103 & 0.3001 \\
\hline \multirow{2}{*}{0.50} & 0.25 & 0.0671 & 3.3128 & 0.0414 & 0.3602 & 0.0671 & 3.3128 & 0.0413 & 0.3486 & 0.0057 & 0.1112 \\
& 0.50 & 0.0358 & 0.3843 & 0.0329 & 0.3610 & 0.0504 & 0.3250 & 0.0314 & 0.3368 & 0.0091 & 0.2887 \\
& 0.75 & 0.0355 & 0.2558 & 0.0420 & 0.2762 & 0.0321 & 0.2738 & 0.0316 & 0.3117 & 0.0066 & 0.1416 \\
\hline
\end{tabular}

$(\lambda=0.2,0.4,0.6,0.8,1.0,1.25,1.5,1.75,2.0$, and 3.0$)$, tardiness factor $(\tau=0.25$ and 0.5$)$, the due date range $(\gamma=$ 0.25 and 0.75 ), and the learning effect (le $=70 \%$ and $90 \%)$. For each situation, 100 replications were randomly generated to yield a total of 16000 instances. Table 3 summarized the performance of the branch-and-bound and SA algorithms. It indicated as follows.

First, the mean and maximum numbers of nodes increased as the job size became larger, as $\lambda$ became smaller, as $\tau$ became smaller, as $\gamma$ became smaller, or as learning effect became strong. The CPU times were observed to increase exponentially as the job size increased. Some of instances could not be solved in the reasonable time. We recorded the number of solvable instances (NSI) if the numbers of nodes were less than $10^{8}$. There were 15,848 solvable instances for all instances. When $(n, \lambda, \tau, \gamma, \mathrm{le})=(14,0.2,0.25,0.25,70)$, the least NSI was 73 which implied that the aforesaid parameters affected the nodes. Second, the accuracy of SA algorithms was assessed, and the results presented in Table 3 indicated that the mean error percentage of $\mathrm{SA}_{5}$ was less than $0.7376 \%$. The performance of error percentage of $\mathrm{SA}_{5}$ trend was not subject to the parameter influenced.

5.3. The Performance of the SA Algorithms for Large Job Size. On evaluating the accuracy of SA algorithms for large job size, we carried out the jobs size $(n=40$ and 80$)$ simulation experiments. The other parameters were taken as $(\lambda=0.2,0.4$, and 0.6$),(\tau=0.25$ and 0.5$),(\gamma=0.25,0.5$, and 0.75$)$, and $(\mathrm{le}=70 \%, 80 \%$, and $90 \%)$. For each situation, 100 replications were randomly generated. For evaluating

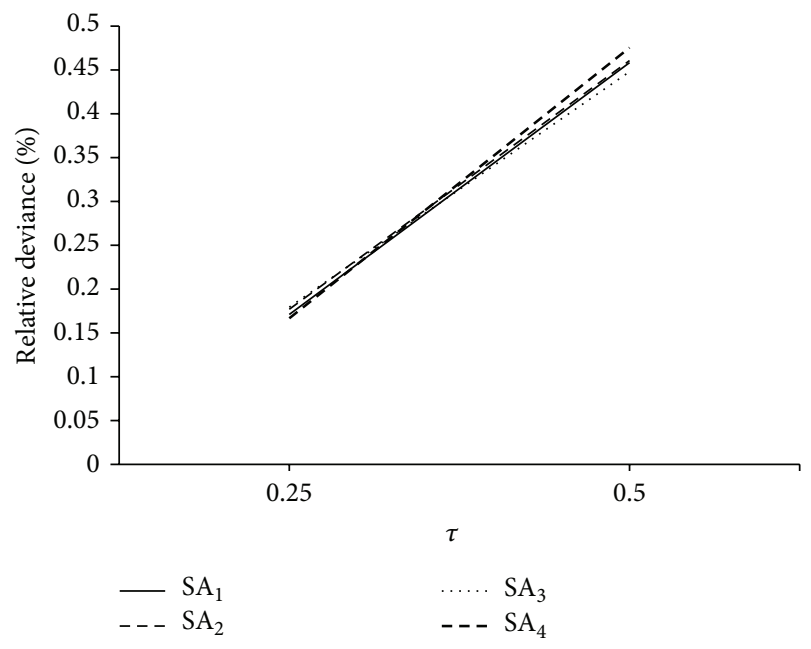

FIGURE 3: The performance of SA algorithm with respect to $\tau$.

the accuracy of SA algorithms, the mean relative deviance percentage was calculated as

$$
\frac{\left(\mathrm{SA}_{i}-\mathrm{SA}_{5}\right)}{\mathrm{SA}_{5}} \times 100 \%,
$$

where $\mathrm{SA}_{i}(i=1,2,3,4)$ was the solution obtained from SA algorithms and $\mathrm{SA}_{5}$ was the smallest value of the objective function obtained from the $i$ th SA algorithms. We get the total average for each $\mathrm{SA}_{i}$ algorithm experimental results as depicted in Figures 1-5. In Figure 4, parameters could slightly 


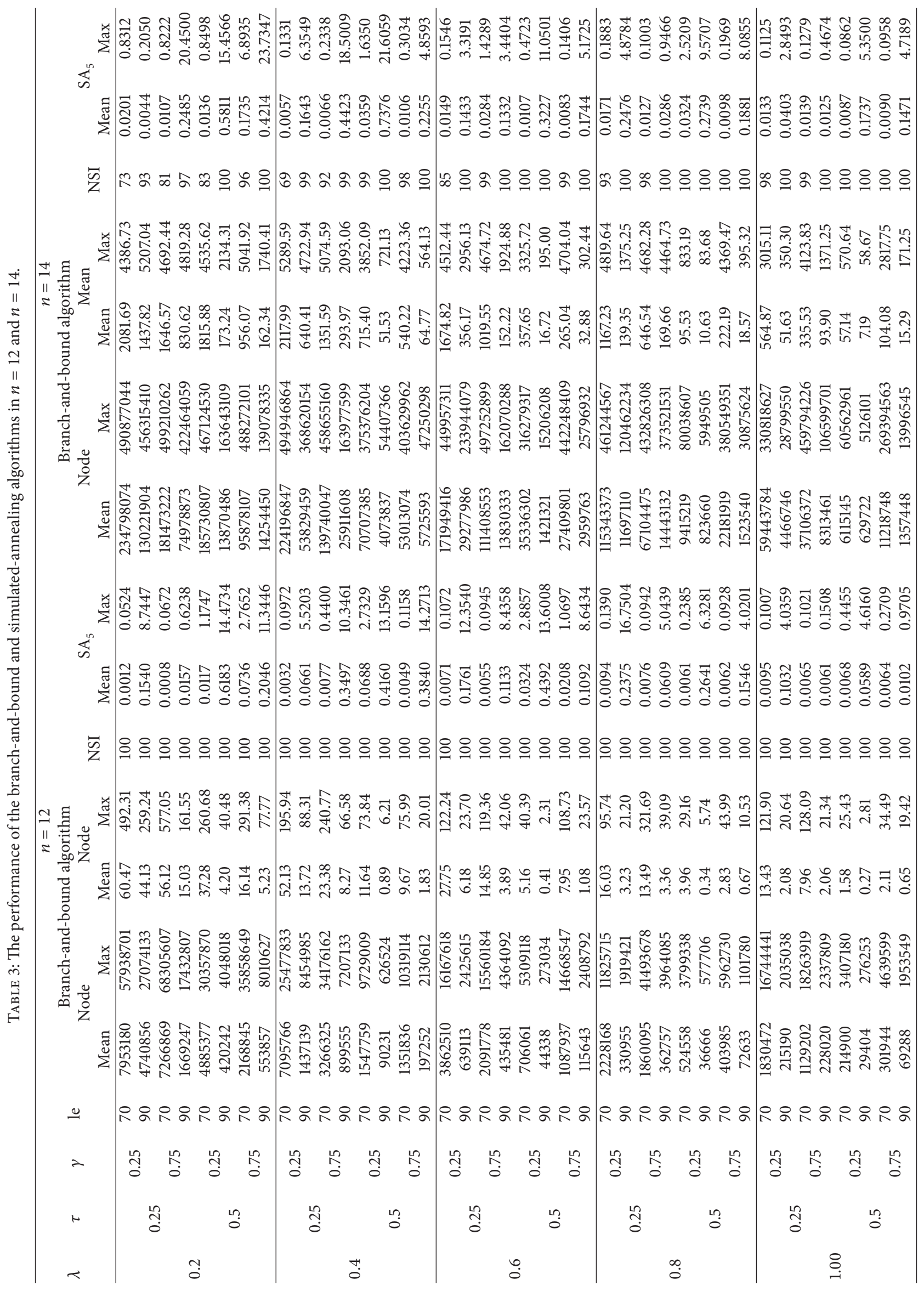




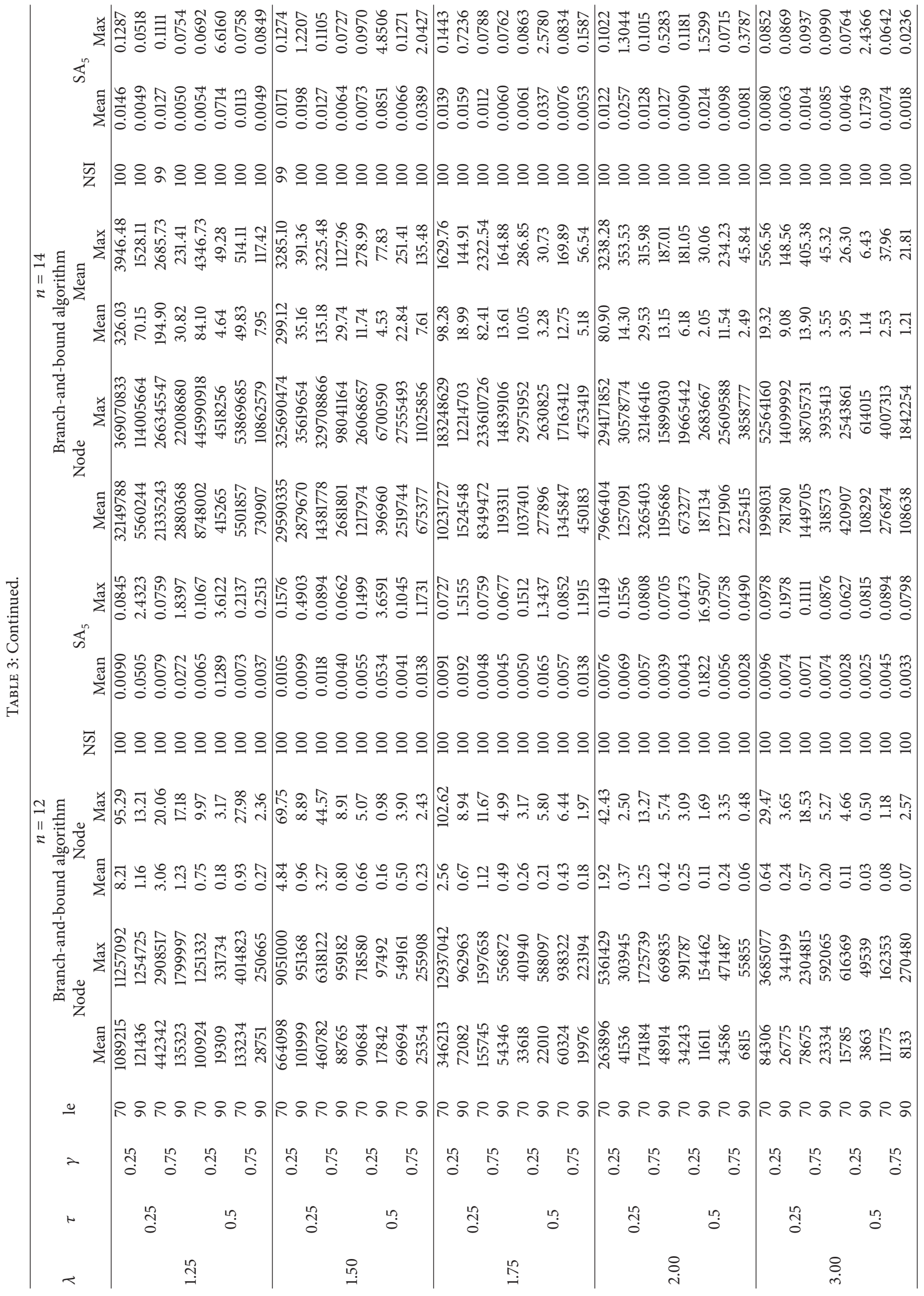




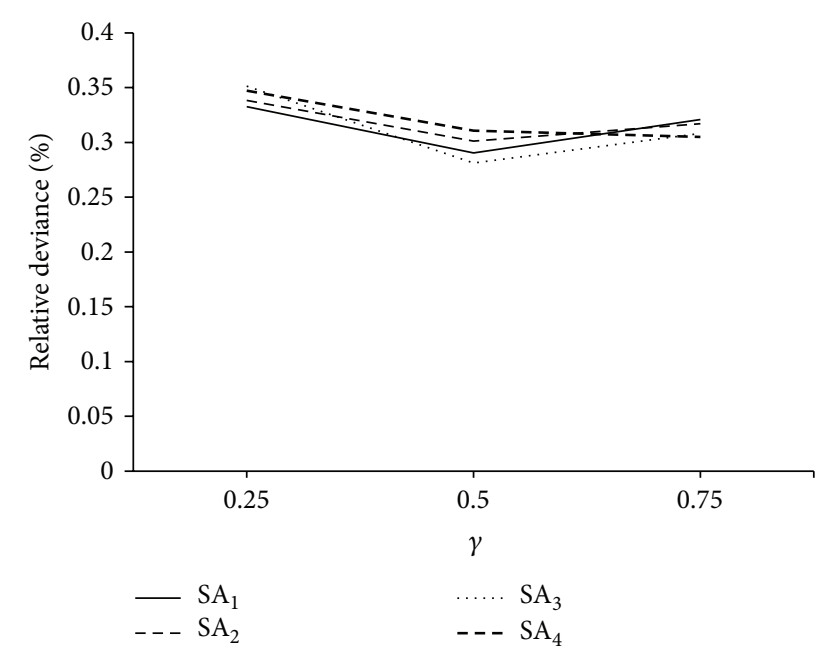

FIGURE 4: The performance of SA algorithm with respect to $\gamma$.

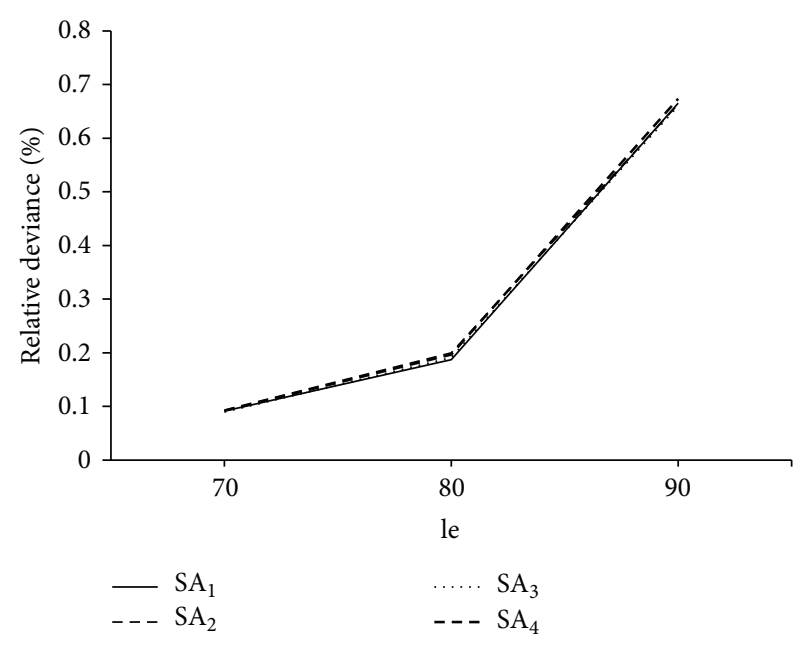

FIGURE 5: The performance of SA algorithm with respect to le.

affect the performance of SAs algorithm. It was observed that there was no significant difference among the performance of SA algorithms. Besides, the CPU times of the SA algorithms were not recorded since they were completed within one second. Thus, the $\mathrm{SA}_{5}$ is recommended for solving the large job size method.

\section{Conclusions}

In this paper, we study the two-agent single-machine scheduling problems in which jobs with arbitrary release times in learning effect condition. The objective is minimizing the total weighted completion time of one agent, subject to an upper bound on the maximum lateness of the second agent. To solve the problem, a branch-and-bound algorithm incorporated with several dominance properties and a lower bound is developed. In addition, simulated-annealing algorithms are also developed to test the accuracy. Computational results show that the branch-and-bound algorithm can find the optimum up to 14 jobs in a reasonable time, and the simulated-annealing algorithm is effective and efficient in obtaining near-optimal solutions. Suggested further research includes considering other optimization criteria or multiobjective optimization to further verify the proposed approach.

\section{References}

[1] D. Biskup, "Single-machine scheduling with learning considerations," European Journal of Operational Research, vol. 115, no. 1, pp. 173-178, 1999.

[2] T. C. E. Cheng and G. Wang, "Single machine scheduling with learning effect considerations," Annals of Operations Research, vol. 98, no. 1-4, pp. 273-290, 2000.

[3] G. Mosheiov, "Scheduling problems with a learning effect," European Journal of Operational Research, vol. 132, no. 3, pp. 687-693, 2001.

[4] G. Mosheiov and J. B. Sidney, "Scheduling with general jobdependent learning curves," European Journal of Operational Research, vol. 147, no. 3, pp. 665-670, 2003.

[5] A. Bachman and A. Janiak, "Scheduling jobs with positiondependent processing times," Journal of the Operational Research Society, vol. 55, pp. 257-264, 2004.

[6] W. C. Lee and C. C. Wu, "Minimizing total completion time in a two-machine flowshop with a learning effect," International Journal of Production Economics, vol. 88, no. 1, pp. 85-93, 2004.

[7] W.-H. Kuo and D.-L. Yang, "Minimizing the total completion time in a single-machine scheduling problem with a timedependent learning effect," European Journal of Operational Research, vol. 174, no. 2, pp. 1184-1190, 2006.

[8] C. Koulamas and G. J. Kyparisis, "Single-machine and twomachine flowshop scheduling with general learning functions," European Journal of Operational Research, vol. 178, no. 2, pp. 402-407, 2007.

[9] D. Biskup, "A state-of-the-art review on scheduling with learning effects," European Journal of Operational Research, vol. 188, no. 2, pp. 315-329, 2008.

[10] J. B. Wang, C. Ng, T. C. E. Cheng, and L. Liu, "Single-machine scheduling with a time-dependent learning effect," International Journal of Production Economics, vol. 111, no. 2, pp. 802-811, 2008.

[11] A. Janiak and R. Rudek, "Experience-based approach to scheduling problems with the learning effect," IEEE Transactions on Systems, Man and Cybernetics A, vol. 39, no. 2, pp. 344-357, 2009.

[12] Y. Yin, D. Xu, K. Sun, and H. Li, "Some scheduling problems with general position-dependent and time-dependent learning effects," Information Sciences, vol. 179, no. 14, pp. 2416-2425, 2009.

[13] M. D. Toksari and E. Güner, "Parallel machine scheduling problem to minimize the earliness/tardiness costs with learning effect and deteriorating jobs," Journal of Intelligent Manufacturing, vol. 21, no. 6, pp. 843-851, 2010.

[14] X. R. Wang, J. B. Wang, W. J. Gao, and X. Huang, "Scheduling with past-sequence-dependent setup times and learning effects on a single machine," The International Journal of Advanced Manufacturing Technology, vol. 48, no. 5-8, pp. 739-746, 2010.

[15] W. C. Lee, C. C. Wu, and P. H. Hsu, "A single-machine learning effect scheduling problem with release times," Omega, vol. 38, no. 1-2, pp. 3-11, 2010.

[16] J.-B. Wang and C. Wang, "Single-machine due-window assignment problem with learning effect and deteriorating jobs," 
Applied Mathematical Modelling, vol. 35, no. 8, pp. 4017-4022, 2011.

[17] C. C. Wu, Y. Yin, and S. R. Cheng, "Some single-machine scheduling problems with a truncation learning effect," Computers \& Industrial Engineering, vol. 60, no. 4, pp. 790-795, 2011.

[18] X. Zhang, G. Yan, W. Huang, and G. Tang, "A note on machine scheduling with sum-of-logarithm-processing-time-based and position-based learning effects," Information Sciences, vol. 187, pp. 298-304, 2012.

[19] Y. Yin, D. Xu, S. R. Cheng, and C. C. Wu, "A generalisation model of learning and deteriorating effects on a single-machine scheduling with past-sequence-dependent setup times," International Journal of Computer Integrated Manufacturing, vol. 25, no. 9, pp. 804-813, 2012.

[20] D.-L. Yang, T. C. E. Cheng, S.-J. Yang, and C.-J. Hsu, "Unrelated parallel-machine scheduling with aging effects and multimaintenance activities," Computers \& Operations Research, vol. 39, no. 7, pp. 1458-1464, 2012.

[21] D. L. Yang, T. C. E. Cheng, and W. H. Kuo, "Scheduling with a general learning effect," The International Journal of Advanced Manufacturing Technology, vol. 67, no. 1-4, pp. 217-229, 2013.

[22] J.-B. Wang, C.-J. Hsu, and D.-L. Yang, "Single-machine scheduling with effects of exponential learning and general deterioration," Applied Mathematical Modelling, vol. 37, no. 4, pp. 22932299, 2013.

[23] J.-B. Wang and J.-J. Wang, "Scheduling jobs with a general learning effect model," Applied Mathematical Modelling, vol. 37, no. 4, pp. 2364-2373, 2013.

[24] T. C. E. Cheng, C. C. Wu, J. C. Chen, W. H. Wu, and S. R. Cheng, "Two-machine flowshop scheduling with a truncated learning function to minimize the makespan," International Journal of Production Economics, vol. 141, no. 1, pp. 79-86, 2013.

[25] K. R. Baker and J. C. Smith, "A multiple-criterion model for machine scheduling," Journal of Scheduling, vol. 6, no. 1, pp. 716, 2003.

[26] A. Agnetis, P. B. Mirchandani, D. Pacciarelli, and A. Pacifici, "Scheduling problems with two competing agents," Operations Research, vol. 52, no. 2, pp. 229-242, 2004.

[27] P. Liu, X. Zhou, and L. Tang, "Two-agent single-machine scheduling with position-dependent processing times," The International Journal of Advanced Manufacturing Technology, vol. 48, no. 1-4, pp. 325-331, 2010.

[28] T. C. E. Cheng, S. R. Cheng, W. H. Wu, P. H. Hsu, and C. C. Wu, "A two-agent single-machine scheduling problem with truncated sum-of-processing-times-based learning considerations," Computers \& Industrial Engineering, vol. 60, no. 4, pp. 534-541, 2011.

[29] D.-C. Li and P.-H. Hsu, "Solving a two-agent single-machine scheduling problem considering learning effect," Computers \& Operations Research, vol. 39, no. 7, pp. 1644-1651, 2012.

[30] W. H. Wu, S. R. Cheng, C. C. Wu, and Y. Yin, "Ant colony algorithms for a two-agent scheduling with sum-of processing times-based learning and deteriorating considerations," Journal of Intelligent Manufacturing, vol. 23, no. 5, pp. 1985-1993, 2012.

[31] A. Baykasoğlu, "Multi-rule multi-objective simulated annealing algorithm for straight and $\mathrm{U}$ type assembly line balancing problems," Journal of Intelligent Manufacturing, vol. 17, no. 2, pp. 217-232, 2006

[32] S. H. Mirsanei, M. Zandieh, M. J. Moayed, and M. R. Khabbazi, "A simulated annealing algorithm approach to hybrid flow shop scheduling with sequence-dependent setup times," Journal of Intelligent Manufacturing, vol. 22, no. 6, pp. 965-978, 2011.
[33] T. S. F. Chan, A. Prakash, H. L. Ma, and C. S. Wong, "A hybrid Tabu sample-sort simulated annealing approach for solving distributed scheduling problem," International Journal of Production Research, vol. 51, no. 9, pp. 2602-2619, 2013.

[34] Y. Yin, S. R. Cheng, T. C. E. Cheng, W. H. Wu, and C. C. $\mathrm{Wu}$, "Two-agent single-machine scheduling with release times and deadlines," International Journal of Shipping and Transport Logistics, vol. 5, no. 1, pp. 75-94, 2013.

[35] S. Kirkpatrick, C. D. Gelatt, Jr., and M. P. Vecchi, "Optimization by simulated annealing," American Association for the Advancement of Science, vol. 220, no. 4598, pp. 671-680, 1983.

[36] N. Metropolis, A. W. Rosenbluth, M. N. Rosenbluth, A. H. Teller, and E. Teller, "Equation of state calculations by fast computing machines," The Journal of Chemical Physics, vol. 21, no. 6, pp. 1087-1092, 1953.

[37] D. Ben-Arieh and O. Maimon, "Annealing method for PCB assembly scheduling on two sequential machines," International Journal of Computer Integrated Manufacturing, vol. 5, no. 6, pp. 361-367, 1992.

[38] C. Chu, "A branch-and-bound algorithm to minimize total flow time with unequal release dates," Naval Research Logistics, vol. 39, no. 6, pp. 859-875, 1992.

[39] M. L. Fisher, "A dual algorithm for the one-machine scheduling problem," Mathematical Programming, vol. 11, no. 1, pp. 229-251, 1976. 


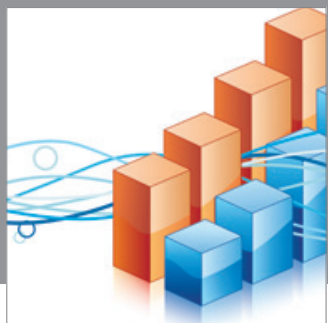

Advances in

Operations Research

mansans

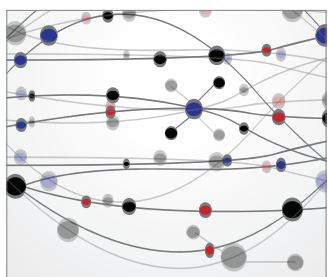

The Scientific World Journal
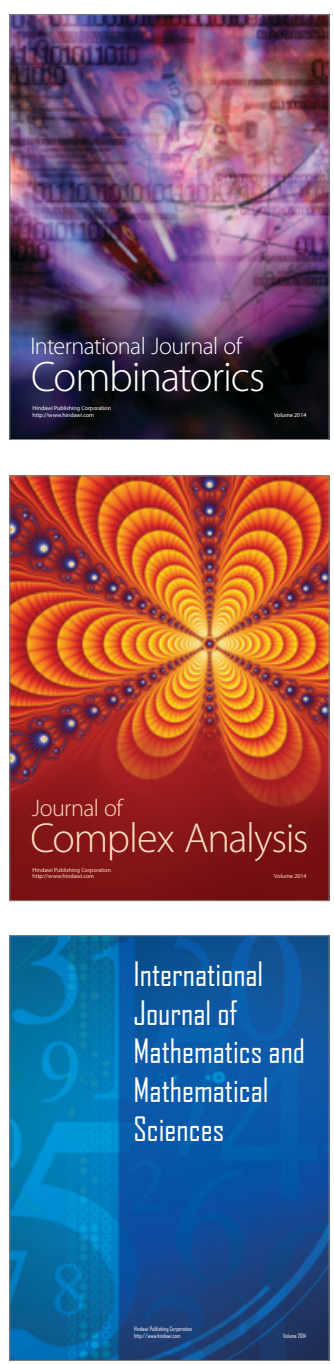
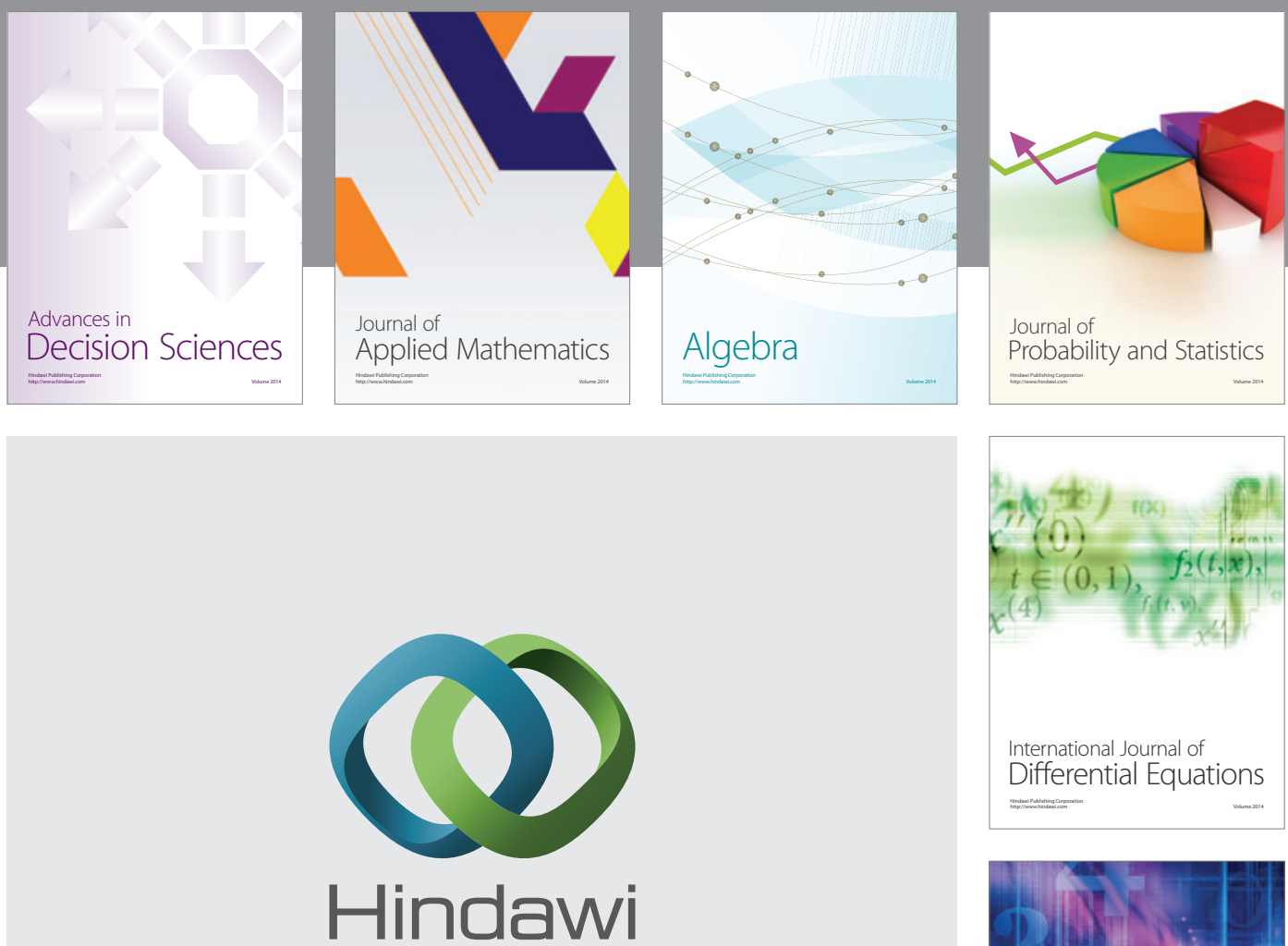

Submit your manuscripts at http://www.hindawi.com
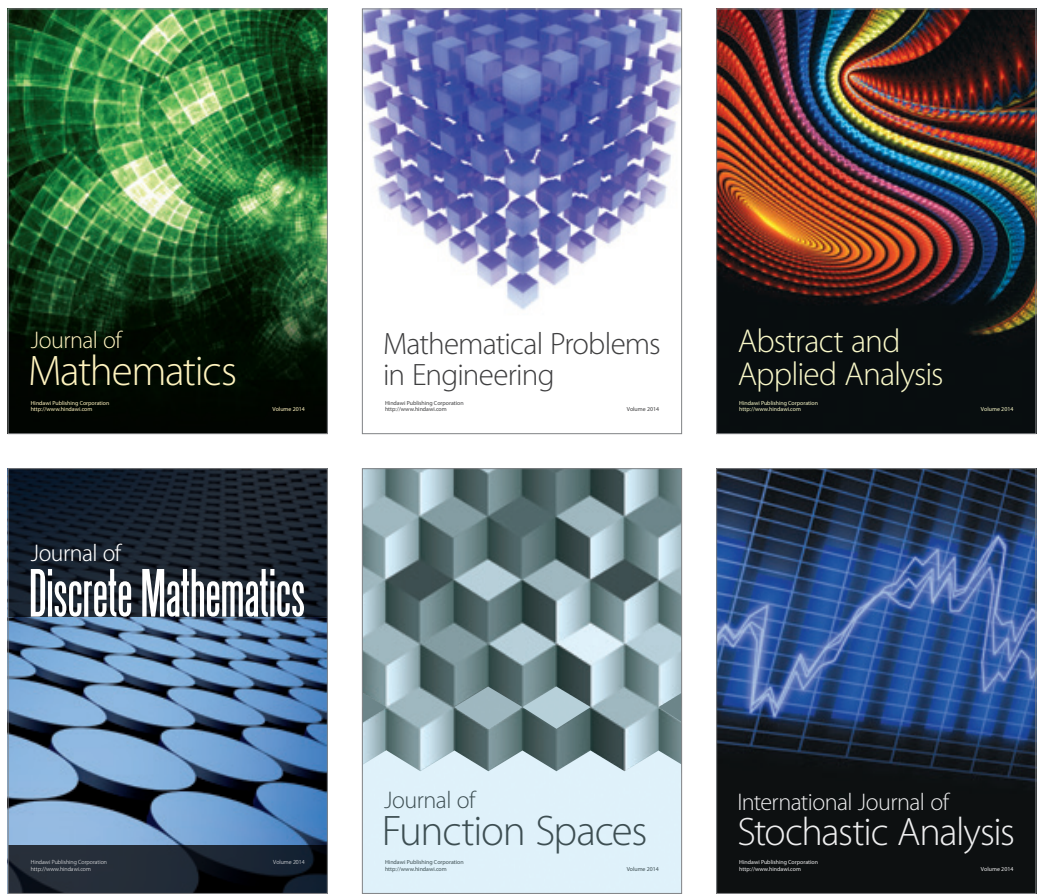

Journal of

Function Spaces

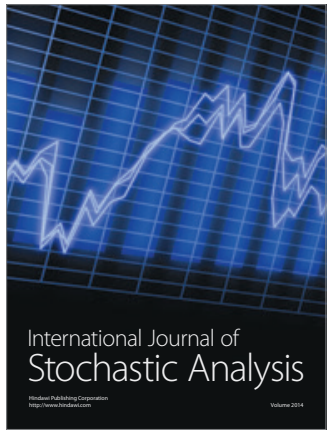

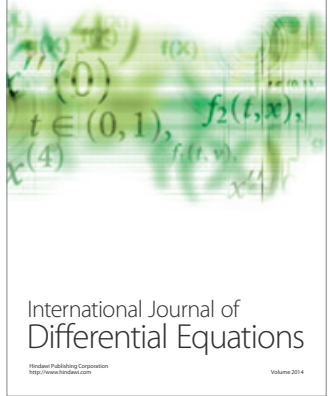
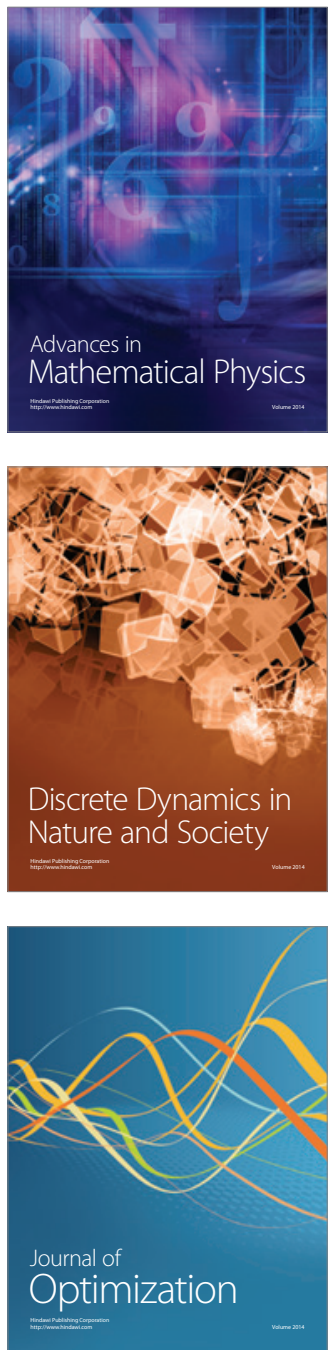